\title{
Comment on: A Pathologist's Approach to Nonimmune Hydrops
}

\author{
Carlo Bellini ${ }^{1}$
}

Received: 7 December 2015/Accepted: 31 March 2016/Published online: 22 April 2016

(C) Society of Fetal Medicine 2016

To the Editor,

I read with interest the excellent and truly valuable review article by WT Parks recently published in the Journal of Fetal Medicine [1], entitled "A Pathologist's Approach to Nonimmune Hydrops" that describes the point of view of the pathologist approaching a fetus or a dead newborn affected by prenatally diagnosed nonimmune hydrops (NIH).

In general, autopsy is recommended in all cases of fetal or neonatal death or pregnancy termination [2]. I fully agree with the author that "determining the cause of the NIH will be the primary question at autopsy" in particular, in cases for which no clinical diagnosis has been made. In Table 1, the author listed chylothorax among the "thoracic" causes of NIH, but neither the table nor the text touches upon lymphatic dysplasia as an important (and often underdiagnosed) cause of NIH. With regard to this issue, I would like to add a brief comment to the article [1]. The role of malformative development or impaired function of the lymphatic system in the fetus leading to hydrops formation has been discussed extensively [3] as also cited by the author. An unacceptably high number of NIH cases remain without a diagnosis, and among them, some degree of lymphatic involvement is extremely likely [4]. The usefulness of immunohistochemical staining techniques to discern between blood and lymphatic microcapillaries and

This comment refers to the article available at doi:10.1007/s40556-015-0055-x.

Carlo Bellini

carlobellini@ospedale-gaslini.ge.it

1 Neonatal Intensive Care Unit, Department of Intensive Care, Gaslini Institute, Largo G. Gaslini, 5, 16147 Genoa, Italy vessels is now well established [4]. We previously recommended [5] the use of CD-31, CD-34, D2-40 (or podoplanin), smooth muscle actin (SMA), anti-LYVE-1 (antibodies against lymphatic endothelium), and VEGFR-3 in the pathological workup of NIH. I would like to underline and reaffirm the importance of immunohistochemical studies in the absence of a definite diagnosis of NIH or, as an alternative in case of a strong suspicion of lymphatic system involvement. I believe the inclusion of accurate immunohistochemical analysis of the lymphatic vessels in all NIH guidelines, might improve the existing value of the article.

\section{References}

1. Parks WT. A Pathologist's Approach to Nonimmune Hydrops. J Fetal Med. 2015;2:143-9.

2. Désilets V, Audibert F. Society of Obstetrician and Gynaecologists of Canada. Investigation and management of non-immune fetal hydrops. J Obstet Gynaecol Can. 2013;35(10):923-38.

3. Bellini C, Hennekam RC. Non-immune hydrops fetalis: a short review of etiology and pathophysiology. Am J Med Genet. 2012;158A(3):597-605.

4. Bellini C, Donarini G, Paladini D, Calevo MG, Bellini T, Ramenghi LA, et al. Etiology of non-immune hydrops fetalis: an update. Am J Med Genet A. 2015;167A(5):1082-8.

5. Bellini C, Rutigliani M, Boccardo F, Campisi C, Fulcheri E, Bellini T, et al. Perinatal deaths and lymphatic system involvement: a diagnostic flow-chart applying immunohistochemical methods. Lymphology. 2011;44(3):131-3. 